\title{
Monte Carlo Study of the Metamagnet Ising model in a Random and Uniform Field
}

\author{
A. Weizenmann, M. Godoy, and A. S. de Arruda \\ Departamento de Física, Universidade Federal de Mato Grosso, 78060-900, Cuiabá, Mato Grosso, Brazil
}

Received on 30 September, 2005

\begin{abstract}
Monte Carlo simulation has been used to determine the phase diagram of a metamagnet Ising model in the presence of a random and uniform magnetic field. The model consists of a spin- $1 / 2$ metamagnet in which the nearest neighbor and next nearest neighbor spin interactions are antiferromagnetic $\left(J_{1}<0\right)$ and ferromagnetic $\left(J_{2}>0\right)$, respectively. We used a bimodal probability distribution for the random magnetic field. We have calculated the staggered magnetization and the fourth-order Binder cumulants in order to obtain the critical points. The phase diagram in the uniform field versus temperature plane presents continuous and first-order transition lines. The phase transition lines, together with the critical and tricritical points, have been obtained for several random field values.
\end{abstract}

Keywords: Random field; Monte Carlo simulation; Tricritical point

\section{INTRODUCTION}

Random field and disordered magnetic systems have been a considerable source of research in the last years [1]. The random field Ising model (RFIM) has been one of the most interesting research topics in theoretical physics (Condensed Matter) in the last fifteen years and it occupies an important position within the physics of disordered systems [2, 3]. Basically, there are two types of disorder in the spin models: disorder bonds (type I) and randomness in the strength of the applied magnetic field (type II). The Spin Glass models, which result from the type I disorder, have been very sucessfull [4]. RFIM, in which the disorder is coupled directly to the external applied magnetic field (type II), has been shown very hard to handle. Although RFIM has deserved many investigations from both experimental and theoretical points of view [3], no significant progress has been achieved to fully understand the nature of the phase transitions and critical behavior. On the other hand, questions such as the lower critical dimension $[5,6]$ and the existence of a static phase transition have already been solved from the theoretical point of view. However, questions such as the existence of the tricritical point [7-11] are still open. The relevance of RFIM is due to the fact that it is the simplest model used to describe the essential physics of a rich class of experimentally accessible disordered systems, which includes: i) structural phase transitions in random alloys [12], ii) commensurate chargedensity-wave systems with impurity pinning [13, 14], iii) binary fluid mixtures in random porous media [15], iv) melting of intercalates in layered compounds such as $T_{i} S_{2}$ [16], v) frustration introduced by the disorder in interacting many body systems, besides explaining several aspects of electronic transport in disordered insulators [17] and vi) systems near the metal-insulator transition $[18,19]$. In the last ten years, the physics of the hysteresis, of the avalanche behavior and of the origin of self-organized criticality [20] have been modelled employing the non-equilibrium behavior of the RFIM. In particular, a new class of problems, such as self-generated glassy behavior, has been studied through the non-disordered model with infinitesimal random field [21]. Recently, random magnetic fields have been considered in metamagnet systems of Ising type. In these systems, only the competition between the ferromagnetic and antiferromagnetic ordering are interesting. However, the application of a random and uniform magnetic field can yield the appearance of new phenomena and a richer critical behavior becomes possible. This is in fact the subject of the present work.

The plan of the paper is as follows. The model and the simulation background are given in section II while the results are discussed in section III.

\section{MODEL AND SIMULATION}

The Hamiltonian model for the spin-1/2 Ising metamagnet can be written as

$$
H=-\sum_{\langle i j\rangle} J_{1} \sigma_{i} \sigma_{j}-\sum_{\langle i k\rangle} J_{2} \sigma_{i} \sigma_{k}-\sum_{i=1}^{N}\left(h-h_{i}\right) \sigma_{i},
$$

where the first and second sum run over all pairs of spin nearest neighbors $(\langle i j\rangle)$ and next nearest neighbors $(\langle i k\rangle)$, respectively, on a simple square lattice with $N$ spins and $\sigma_{i}= \pm 1$. $J_{1}<0$ (antiferromagnetic) represents the nearest neighbor spin interactions and $J_{2}>0$ (ferromagnetic) represents the next nearest neighbor spin interactions. $h$ is the strength of the external uniform magnetic field and the random magnetic fields $h_{i}$ in the last sum are governed by the bimodal distribution

$$
P\left(h_{i}\right)=\frac{1}{2}\left[\delta\left(h_{i}-h_{r}\right)+\delta\left(h_{i}+h_{r}\right)\right]
$$

where $h_{r}$ is the strength of the random field.

To study this system we employed Monte Carlo simulation techniques [22]. We consider a square lattice of linear size $L$, with values of $L$ ranging from $L=16$ to $L=128$, and we applied periodic boundary conditions. We prepared the system with the spins randomly distributed on the lattice. Each trial change of a spin state on the lattice is accepted according to the Metropolis prescription, $\omega\left(\alpha \rightarrow \alpha^{\prime}\right)=$ $\min [1, \exp (-\beta \Delta E)]$, where $\Delta E$ is the local energy change $\left(E_{\alpha^{\prime}}-E_{\alpha}\right)$ resulting from changing the state of a random selected spin from $\alpha$ to $\alpha^{\prime}$ state, and $\beta=1 / k_{B} T$. To reach the 
equilibrium state we take, for guarantee, at least $2 \times 10^{5} \mathrm{MCs}$ (Monte Carlo steps) for all the lattice sites we studied. Then, we take more $3 \times 10^{5} \mathrm{MCs}$ to estimate the average values of the quantities of interest. Here, 1 MCs means $L^{2}$ trials for change the state of a spin of the lattice.

We calculated the sublattice magnetizations per spin

$$
m_{1}=\left[\frac{1}{N_{1}}\left\langle\sum_{i \in 1} \sigma_{i}\right\rangle\right]_{h_{r}},
$$

and

$$
m_{2}=\left[\frac{1}{N_{2}}\left\langle\sum_{i \in 2} \sigma_{i}\right\rangle\right]_{h_{r}} .
$$

The transition lines of the phase diagram were obtained from the staggered magnetization, defined as $m_{S}=\left(m_{1}-m_{2}\right) / 2$. We also calculated the reduced fourth-order Binder cumulants [23]

$$
U_{L}=1-\frac{\left[\left\langle m_{s}^{4}\right\rangle\right]_{h_{r}}}{3\left[\left\langle m_{s}^{2}\right\rangle^{2}\right]_{h_{r}}},
$$

associated with the staggered magnetization. From the crossing point of the cumulants for different lattice sizes we determined the critical points of the model [23]. In the above expressions $[\cdots]_{h_{r}}$ denotes the average over the disorder and $\langle\cdots\rangle$ denotes the thermal average. The average over the disorder was done using $N_{\text {samp }}=100$ independent samples for lattices in the range $16 \leq L \leq 128$.

\section{RESULTS}

We present in Fig. 1 the phase diagram of the model in the plane external uniform field $h$ versus temperature $T$, for three selected values of the random field $h_{r}$. The phase diagram displays continuous phase transition lines which end at a tricritical point at low temperatures. The solid curves joining the square are continuous phase transition lines while the dashed lines represent first-order transitions. Circles represent the positions of the tricritical points. The points in the continuous transition lines were obtined by considering the common point where the cumulants for different lattice sizes cross. As an example, we show in Fig. 2 a typical case for $h_{r}=0$ and $h=1.0$. On the other hand, the first-order transition lines were estimated by observing the discontinuity in the staggered magnetization at the transition point. An example of this is given in Fig. 3. We found the tricritical points for the following random fields (see Fig. 1): (a) $h_{r}=0.0$ $\left(T_{t}=2.52, h_{t}=3.90\right)$, (b) $h_{r}=0.5\left(T_{t}=2.40, h_{t}=3.80\right)$ and (c) $h_{r}=1.0\left(T_{t}=2.30, h_{t}=3.30\right)$. For $h=0$ and different values of $h_{r}$ the phase diagram presents different values for the critical temperature. The result shown in (Fig. 1) is qualitatively similar to that obtained by the use of a mean-field calculation [24].

In Fig. 3 we show the behavior of the staggered magnetization $m_{s}$ as a function of the external uniform field $h$. For

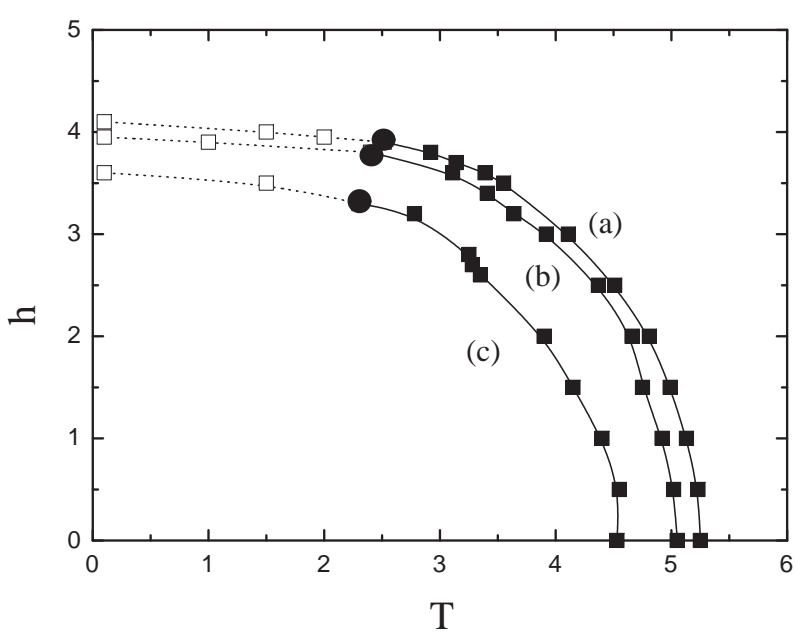

FIG. 1: Phase diagram in the plane uniform field $h$ versus temperature $T$ for three different values of the random field $h_{r}$. The solid lines joining the full squares represent continuous transitions while dashed lines joining the open squares represent first-order transitions. Full circles represent the positions of the tricritical points. (a) $h_{r}=0.0$ $\left(T_{t}=2.52, h_{t}=3.90\right)$, (b) $h_{r}=0.5\left(T_{t}=2.40, h_{t}=3.80\right)$ and (c) $h_{r}=1.0\left(T_{t}=2.30, h_{t}=3.30\right)$. The erros bars are less than the size of the data points.

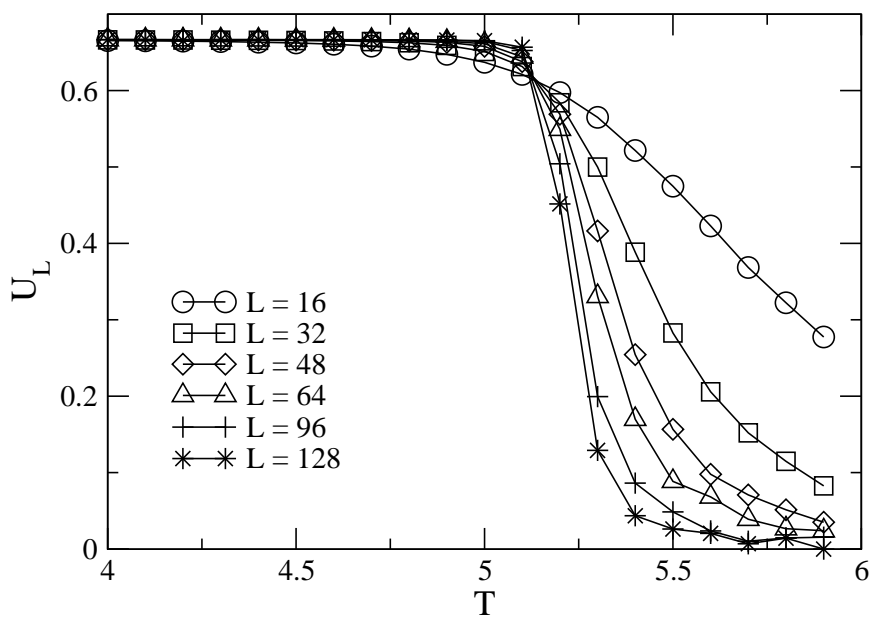

FIG. 2: Fourth-order Binder cumulants $U_{L}$ versus temperature $T$, for various lattice sizes as indicated in the figure and for the point $h_{r}=0$, $h=1.0$. The erros bars are less than the size of the data points.

the set values of temperatures $T=1.5, T=2.0$, and for a random field $h_{r}=0.0$, we observe that the staggered magnetizations are discontinuous, clearly indicating the existence of a first-order phase transition. On the other hand, for the temperature $T=3.0$, we can observe that a staggered magnetization vanishes continuously with the increase of the uniform field 


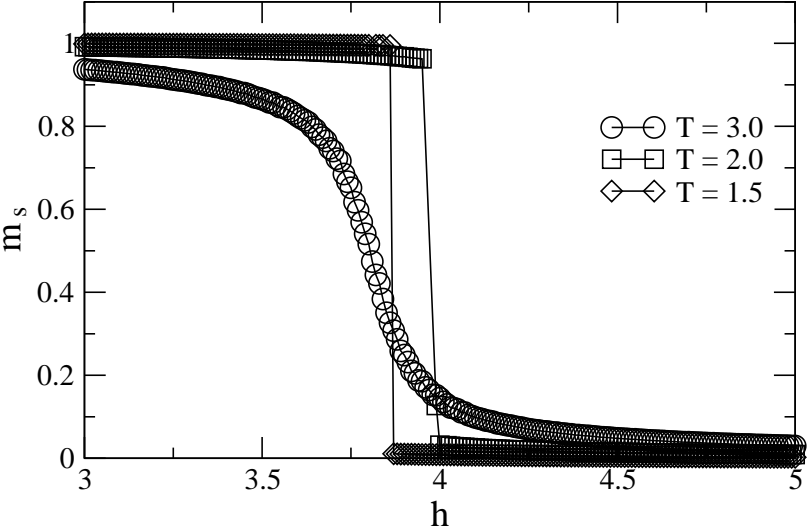

FIG. 3: Staggered magnetization as a function of the external uniform field $h$, for several values of the temperature $T$ as indicated in the figure for $h_{r}=0.0$. $h$, indicating the existence of a continuous phase transition. From the crossing of the analytical continuation of the firstand second-order lines we estimate the location of the tricritical points shown in Fig. 1.

In summary, the present Monte Carlo simulations for a metamagnet Ising model in a random and uniform field show that the phase diagram in the plane uniform field $h$ versus temperature present continuous and first-order transition lines separated by tricritical points.

\section{Acknowledgments}

The authors acknowledge the financial support given by the Brazilian agencies CAPES, CNPq and FAPEMAT.
[1] D. P. Belanger, Braz. J. Phys. 30682 (2000).

[2] D. P. Belanger, in Spin Glasses and Random Fields, edited by A. P. Young (World Scientific, Singapore, 1998); R. J. Birgeneau, J. Magn. Magn. Mater. 177, 1 (1998).

[3] Y. Shapir, in Recent Progress in Random Magnets, edited by D. H. Ryan (World Scientific, Singapore, 1992), pp. 309-334.

[4] J. R. L. de Almeida and D. J. Thouless, J. Phys. A 11, 983 (1978).

[5] J. Z. Imbrie, Phys. Rev. Lett. 531747 (1984).

[6] Y. Imry and S. K. Ma, Phys. Rev. Lett. 351399 (1975).

[7] A. S. de Arruda, W. Figueiredo, R. M. Sebastianes, and V. K. Saxena, Phys. Rev. B 394409 (1989).

[8] A. S. de Arruda and W. Figueiredo, Mod. Lett. B $11(21,22)$ 973 (1997).

[9] D. F. de Albuquerque and A. S. de Arruda, Physica A 31613 (2002).

[10] D. C. Mattis, Phys. Rev. Lett. 553009 (1985).

[11] A. Aharohy, Phys. Rev. B 183318 (1978).

[12] J. R. Childress and C. L. Chien, Phys. Rev. B 43, 8089 (1991).

[13] D. S. Fisher, Phys. Rev. Lett. 50, 1486 (1983).
[14] D. S. Fisher, Phys. Rev. B 31, 1396 (1985).

[15] J. V. Maher, W. I. Goldburg, D. W. Pohlm and M. Lanz, Phys. Rev. Lett. 53, 60 (1984).

[16] R. M. Suter, M. W. Shafer, P. M. Hornm and P. Dimon, Phys. Rev. B 26, 1495 (1982).

[17] A. L. Efros and B. L. Shklovskii, J. Phys. C 8, L49 (1975).

[18] T. R. Kirkpatrick and D. Belitz, Phys. Rev. Lett. 73, 862 (1994).

[19] A. A. Pastor and V. Dobrosavljević, Phys. Rev. Lett. 83, 4642 (1999).

[20] A. A. Pastor, V. Dobrosavljević, and M. L. Horbach, Phys. Rev. B 66014413 (2002).

[21] M. Mezard, Physica A 265, 352 (1999).

[22] D. P. Landau and K. Binder, A Guide to Monte Carlo Simulations in Statistical Physics (Cambridge University Press, United Kingdom, 2000).

[23] K. Binder and D. W. Heermann, Monte Carlo Simulation in Statistical Physics. An Introduction, 3rd ed. (Springer, Berlin, 1997).

[24] S. Galam, C. S. O. Yokoi, and S. R. Salinas, Phys. Rev. B 57, 8370 (1998). 